\title{
Historic HOLC Redlining in Indianapolis and the Legacy of Environmental Impacts
}

\author{
Donovan Moxley \& Burnell Fischer ${ }^{1}$ \\ ${ }^{1}$ bufische@indiana.edu
}

Redline mapping was adopted by the Home Owner's Loan Corporation (HOLC) in the 1930s to help assess its lending risk based on observable characteristics in urban neighborhoods across the United States, but the practice has been criticized for evident racial discrimination in addition to financial risk assessment. Researchers have demonstrated that modern urban tree canopy cover is lower in the historically redlined zones of four US cities. This article focuses on Indianapolis, Indiana, where a HOLC map was developed in 1937. Analysis using a geographic information system (GIS) was conducted in order to detect evidence of ecological legacies of redlining. Using this method, evidence of relatively high-intensity development, low greenspace and forest cover, and disproportionately high incidences of brownfield sites, Superfund sites, industrial waste sites, and Interstate highways were detected in historically redlined zones in Indianapolis. While opportunities for further and more detailed research are considered, the method employed here should be developed such that it is replicable for other cities with redlining histories in Indiana and other surrounding states with comparable spatial datasets. This example protocol can be adopted and refined by various stakeholders to establish where the need may exist for programmatic solutions and to better inform policy recommendations.

Keywords: Redlining, Legacy, Ecology, Zoning, Indianapolis, GIS, Protocol

\section{INTRODUCTION}

The Home Owners' Loan Corporation (HOLC) helped the federal government to offer balanced financing to one million borrowers in the mid-1930s who had lost their homes or faced foreclosure due to backloaded loans taken out before the Great Depression (Hillier 2003). The HOLC produced maps that classified risk of loan defaults in cities across the United States. These maps accounted for, among other socioeconomic factors, the ethnic and racial profiles (Figure 1) described for each mapped zone (University of Richmond). In a formalization of pre-existing practices, zones of the lowest grade (D) were shaded red, considered "hazardous" financial risks and residents routinely were denied loans (Hillier 2003). Evidence of a correlated ecological legacy emerged from a modern tree canopy cover analysis of four U.S. cities Durham, NC; Fresno, CA; Pittsburgh, PA; and Portland, OR. Specifically, canopy cover steadily decreased moving from Grade A through Grade D

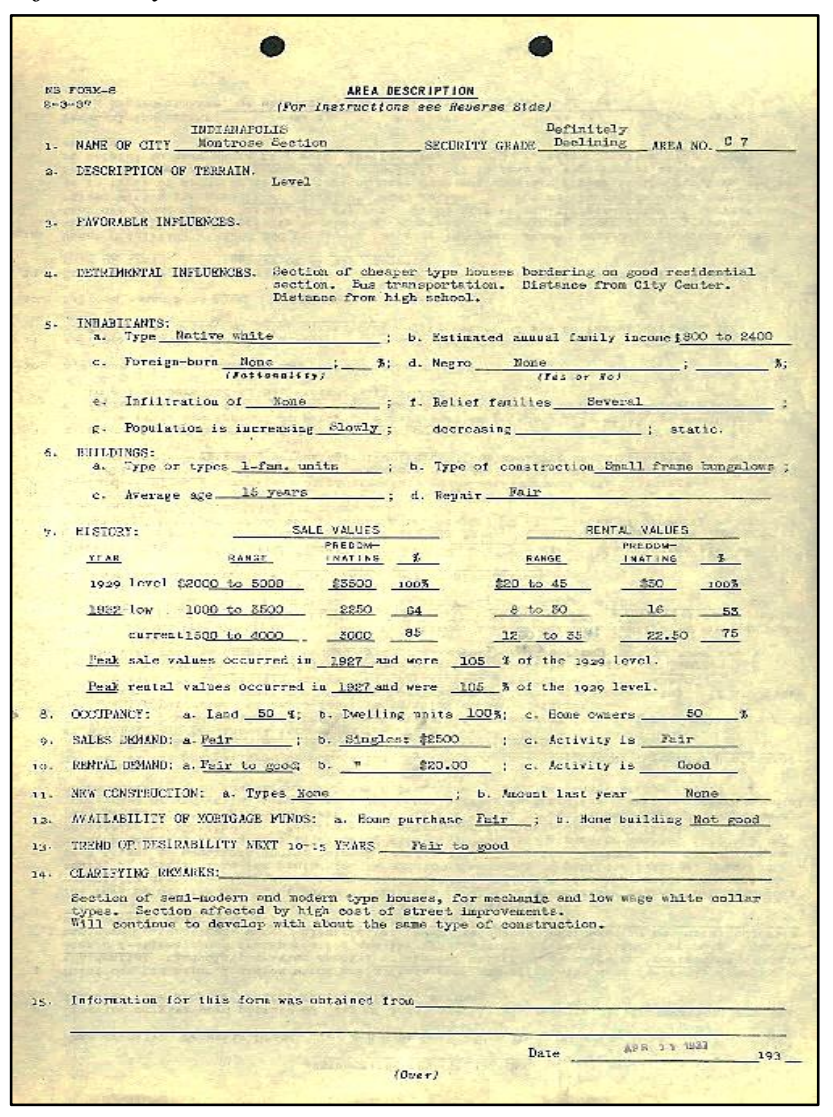

Figure 1. Digital scan of the Area Description report for Zone C7, dated April 11, 1937. Reports of this type remain viewable for most HOLC zones in the city. Source: University of Richmond. 


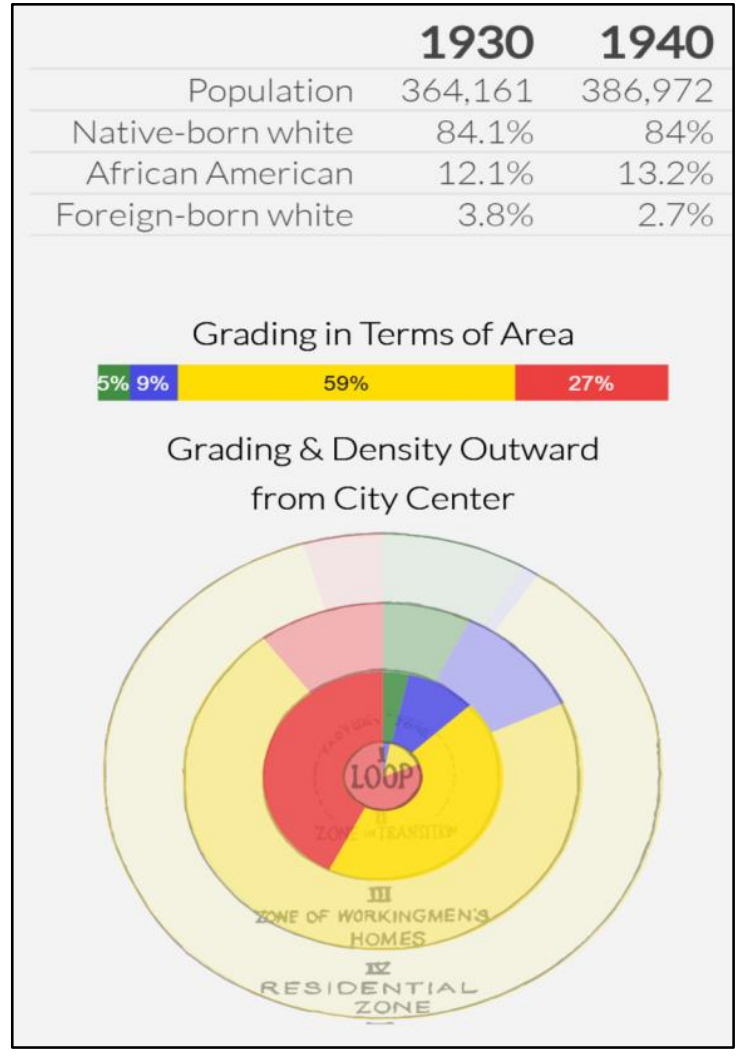

Figure 2. Historical demographic and HOLC grading overview for Indianapolis, Indiana. Source: University of Richmond.

zones (Cooper et al. 2016). Tree cover importantly contributes ecosystem services at various spatial scales (Livesley et al. 2016).

Indianapolis, Indiana, was one of 192 U.S. metropolitan areas for which the University of Richmond has provided digitally scanned historical redlining maps (Nelson et al. 2019). The state capital, Indianapolis, outsized each of the other redlined Indiana cities by three times or more. These other cities included Evansville, Fort Wayne, Gary, Muncie, South Bend, and Terre Haute. The 1930s were a decade of growth for the city Indianapolis (University of Richmond). Both the overall population and proportion of African Americans increased during that decade (Figure 2), and each of these measures has more than doubled over the course of the seven decades to follow (US Census Bureau 2015). Over time, as industry, economy, policy, and infrastructure evolved in Indianapolis, environmental burdens were added to the city that persist to various extents today.
The HOLC loan security map for the city of Indianapolis dates from 1937 (Figure 3). The boundaries of this map were the area of interest for this article. The goals are to detect evidence of any ecological legacies that correlate to Indianapolis's redlining history and, in doing so, to develop techniques and presentation of results that are adaptable to other cities. We present a detailed methodology which we apply to the city of Indianapolis, but which can also serve as an example for similar projects to explore redlining legacies across the U.S. with relative ease.

\section{METHODS}

A digital copy of the HOLC map for Indianapolis (Figure 2) was captured from the University of Richmond's web project, Mapping Inequality: Redlining in New Deal America (University of Richmond). Using the software ArcMap 10.4, the HOLC scan was georeferenced using six control points at the edges and interior of the map. Then, from the editor toolbar, the "create features" process was used to trace polygons over each of the 71 zones that appeared in the map. As features were drawn, identifying information (grade and zone number) was assigned in the attribute table. In the properties of this new feature class, the symbology was set to display each grade with its historical green, blue, yellow, or red shading. While the attribute table was still open, a new field was added to the table and the Calculate Geometry option was selected so that the surface area would be attributed to each zone. This digitized map layer (Figure 4) was saved as a shapefile and regarded as the official area of interest for all subsequent analysis.

All additional map layers were also freely available on the Web, hosted by the Indiana Geographic Information Council among their IndianaMAP resources. The first IndianaMAP layer downloaded was the National Land Cover Database (NLCD) raster layer (USGS 2015). This resource was chosen for its accessibility. The prior study of tree canopy cover in historically redlined cities benefited from 1-meter raster data produced by the Environmental Protection Agency for a total of 16 


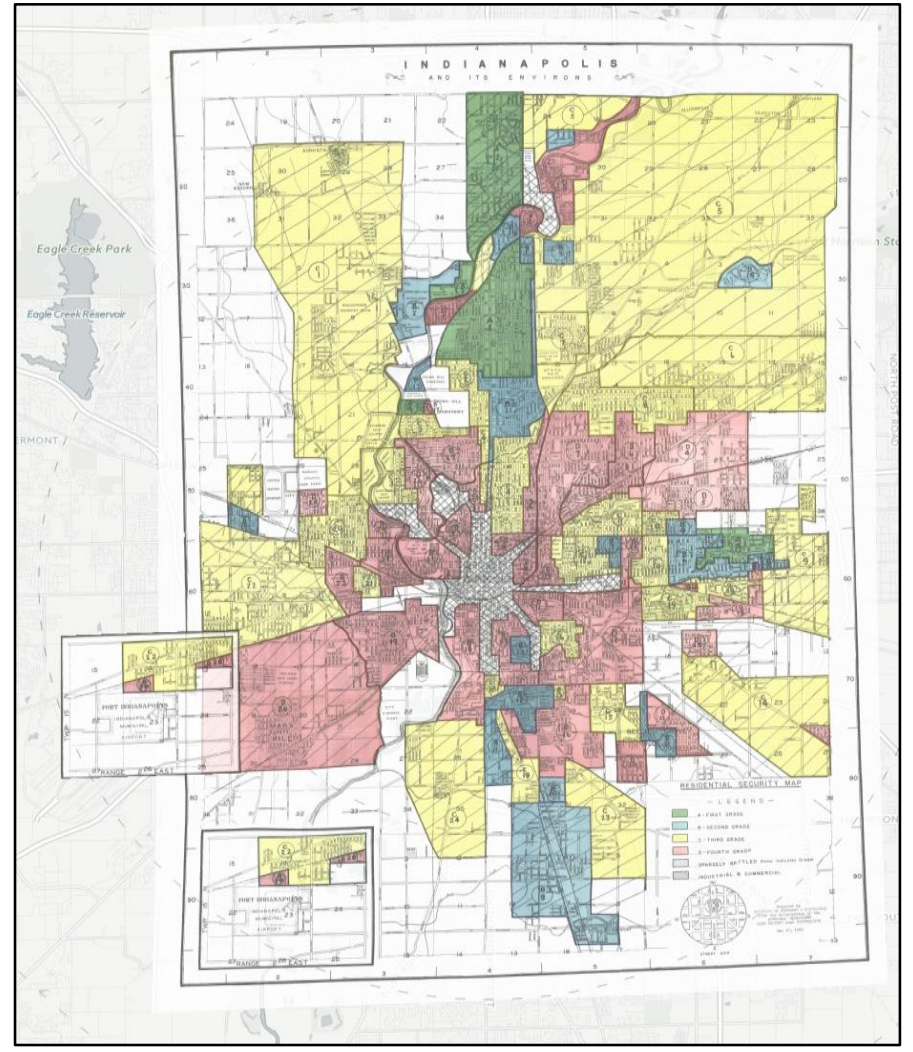

Figure 3. Digital scan of the 1937 HOLC redlining map for Indianapolis, Indiana. Source: University of Richmond.

cities using a Normalized Difference Vegetation Index (NDVI) technique (Cooper et al. 2016).

Though the NDVI protocol is technically replicable, the computing power demanded by ArcMap to complete it for Indianapolis proved overwhelming. Thus, in the interest of producing a more easily adapted example protocol, this article focuses on the less precise NLCD raster.

This raster was an image (.tif file) showing land cover classes from the year 2011 for the entire state of Indiana on a 30-meter grid. From ArcToolbox, the Raster to Polygon tool was selected from the Conversion Tools menu, resulting in a polygon layer which could be trimmed to the area of interest. Within the Analysis Tools, the Intersect tool was chosen from the Overlay menu. Both the HOLC polygon and land cover polygon layers were selected as input features, so the output feature class combined attributes from both layers and only displayed land cover as it occurred within the borders of the HOLC zones.

Of the numerous available layers on IndianaMAP, four modern variables were downloaded for this pilot project. Brownfield sites (IDEM 2015a), Superfund sites (IDEM 2015b), Industrial waste sites (IDEM 2015c), and Interstate highway mile markers (INDOT 2015) were pertinent to the research goals. Each has implications for nearby environmental and ecological health. These layers also share common formatting (each is a vector layer comprised of point features), which allowed for virtually identical process steps as each was imported into ArcMap.

Separately, each layer was processed using the Intersect tool so that only points situated within the HOLC zones would be displayed on the map. This brought a total of five modern environmental factors into the spatial boundaries of the historic HOLC zones. Maps were exported that display each of these intersections.

Data for each of these newly constructed layers included attributes from each of the input layers. For example, each feature in the newly

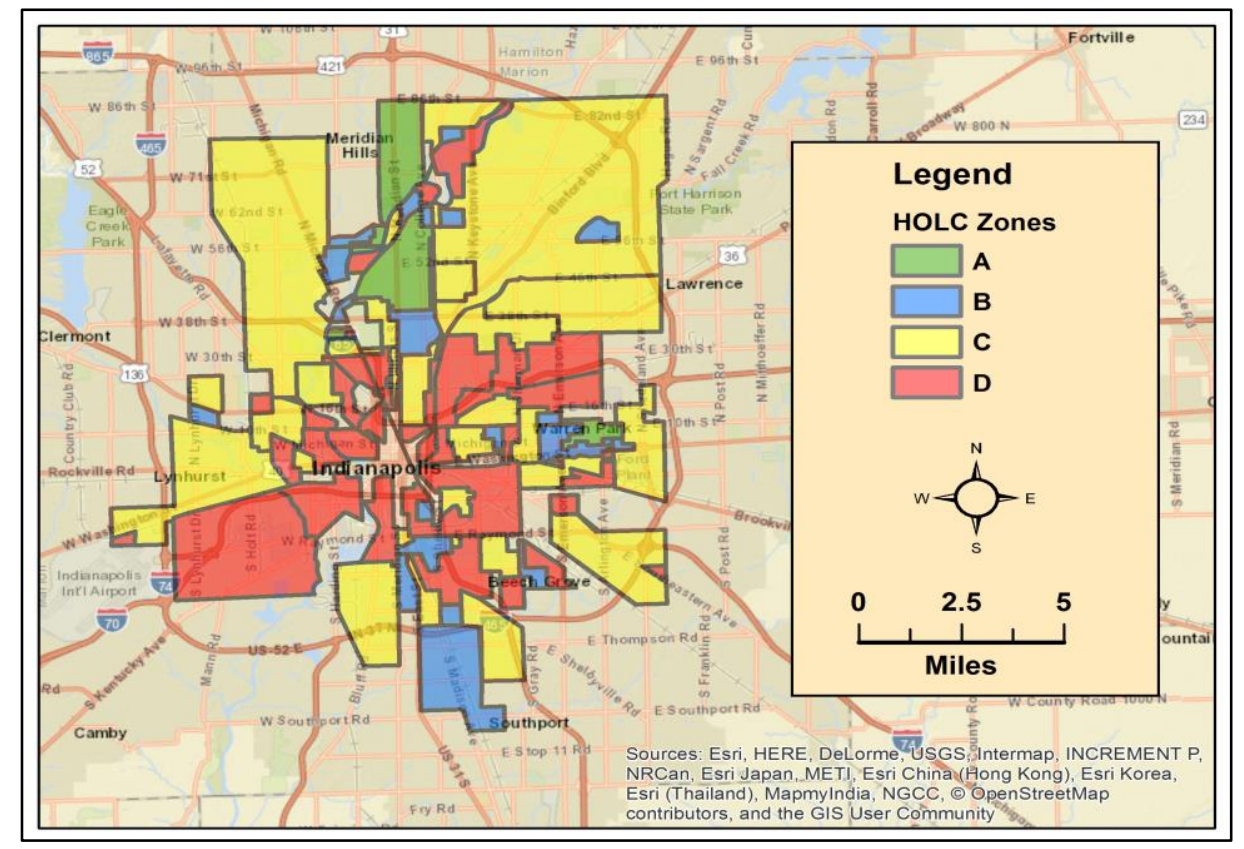

Figure 4. Lending security zones of the HOLC loan risk map (1937), laid over a modern-day street map of Indianapolis, Indiana. 
clipped brownfield layer included all original attributes from the default brownfield layer as well as the respective HOLC attributes, including the specific zone class and number where it could be found. The Calculate Geometry feature was used to determine the area of each polygon in square meters.

All non-spatial data summaries and analyses were completed in Microsoft Excel. Attribute tables for all combined layers were exported from ArcMap using the Table to Excel tool (found in the Conversion Tools of ArcToolbox under the Excel heading), which created spreadsheet (.xlsx) files. The attributes from each layer that intersected with the HOLC polygons were exported as tables into Microsoft Excel (.xlsx) files for data analysis. All other calculations, including unit conversions and summing of land areas, were completed in Excel. All tabular summaries were constructed and formatted in Excel.

\section{RESULTS}

A majority of the land area assigned in 1937 was designated Grade C, though more zones were assigned to Grade D (i.e., "redlined") than any other. In total, 27 percent of the 138.9 square mile area of interest was redlined (Table 1). A majority of the HOLC zones are situated inside the Interstate-465
Table 1. Summary of 1937 HOLC zones in Indianapolis, Indiana, based on geometry calculations of vectors drawn in ArcMap 10.4 and summarized in Microsoft Excel.

\begin{tabular}{|l|cccc|c|}
\hline Grade & A & B & C & D & TOTAL \\
\hline Zone Count & 5 & 17 & 23 & 26 & 71 \\
Area, sq. mi. & 7.5 & 12.2 & 81.8 & 37.4 & 138.9 \\
\hline
\end{tabular}

loop that, decades after this instance of redlining, has come to encircle most of Indianapolis and Marion County. Most of the Grade A land area was found in the north part of the city, near the Meridian Hills neighborhood. Neighborhoods gaining Grade B designation were scattered throughout the city, while Grade $\mathrm{C}$ designations were common on the outer edges of the HOLC map. The redlined zones were especially common in the central core of the city and nearby to the south. On average, HOLC zones in Indianapolis were less than 2 square miles each; however, the largest (Zone C5, northeast corner of the map) was greater than 25 square miles (Figure 5).

Current land cover imagery (2011) reveals that most of the area mapped by the HOLC is now developed. With each successively higher grade, the proportion of developed land cover decreased while the proportion of greenspace increased. In addition to having the greatest amount of developed land cover overall, redlined zones had higher percentages of medium- and highintensity development. In a comparison of extreme categories, there was greater than a five-fold difference between greenspace coverage in Grade A versus Grade D zones, while forest cover per acre was more than seven times greater in Grade A versus Grade D zones (Table 2, Figure 5). The disparities appear less dramatic when the redlined zones are compared to"nonredlined" zones (i.e., Grades A, B, and $\mathrm{C}$ taken together), but the redlined citizens still enjoy less than half the greenspace and forest cover per area (Table 3, Figure 5) than the rest of the neighborhoods. 
Table 3. Land cover (2011) in non-redlined (Grades A-C) versus redlined (Grade D) zones designated in the HOLC lending security map (1937).

\begin{tabular}{|l|cc|c|}
\cline { 2 - 3 } \multicolumn{1}{c|}{} & \multicolumn{2}{c|}{ Percentage by HOLC Zone } & \multicolumn{1}{c|}{} \\
\hline Land Cover & A B C & D & All HOLC \\
& (6347 Acres) & (3018 Acres) & (9364 Acres) \\
\hline Open Water & 0.6 & 0.8 & 0.7 \\
Developed - Open & 22.2 & 19.0 & 21.2 \\
Developed - Low Intensity & 32.0 & 29.2 & 31.1 \\
Developed - Medium Intensity & 31.9 & 36.0 & 33.2 \\
Developed - High Intensity & 9.4 & 13.3 & 10.7 \\
Barren & 0.0 & 0.0 & 0.0 \\
Forest - Deciduous & 2.5 & 1.0 & 2.0 \\
Forest - Evergreen & 0.1 & 0.0 & 0.1 \\
Shrub & 0.2 & 0.0 & 0.1 \\
Herbaceous & 0.0 & 0.0 & 0.0 \\
Hay/Pasture & 0.0 & 0.0 & 0.0 \\
Cultivated Crops & 0.1 & 0.0 & 0.1 \\
Wetlands - Woody & 0.1 & 0.1 & 0.1 \\
Wetlands - Herbaceous & 0.7 & 0.6 & 0.7 \\
\hline Percent Developed & 95.6 & 97.5 & 96.2 \\
Percent Agricultural & 0.1 & 0.1 & 0.1 \\
Percent Green Space & 3.6 & 1.7 & 3.0 \\
\hline Percent Forest & 2.6 & 1.0 & 2.1 \\
\hline
\end{tabular}

\section{DISCUSSION}

Land cover analysis reveals that historically redlined zones have experienced more intense development than other parts of the city. Unless some of the development in historically redlined areas could be reversed, there is such a high rate of developed lands in Grade D zones that achieving forest coverage comparable to the Grade A or B zones will be virtually impossible.

In this analysis, the use of the available National Land Cover Database layer is likely to detect urban tree canopy only where it occurs in relatively large patches, due to the 30-meter grid comprising the imagery. Thus, while this trend of higher canopy cover for higher HOLC grades was consistent with the results in four cities previously analyzed (Cooper et al. 2016), important differences exist between the two approaches to measuring forest percent of modern brownfields in the study area of Indianapolis were found in zones that were redlined in 1937 (Figure 6a). The Superfund sites registered to the study area included waste handling and storage facilities which went into operation in the 1950s1970s (US EPA), and all four were in historically redlined areas (Figure $6 b)$. Industrial waste sites were located all over the city, but $49.1 \%$ of them were found in redlined zones (Figure 6c), although redlined zones only made up 27 percent of land area (Figure 3, Table 1). Two stretches of interstate highway cut across the city. Of the I-65 and I-70 mile markers found within the study area, most $(69.2 \%)$ were found to be situated in the historically redlined zones (Figure 6d). All of these environmental burdens were disproportionately placed upon the citizens of redlined areas in the decades that followed the creation of the HOLC map.

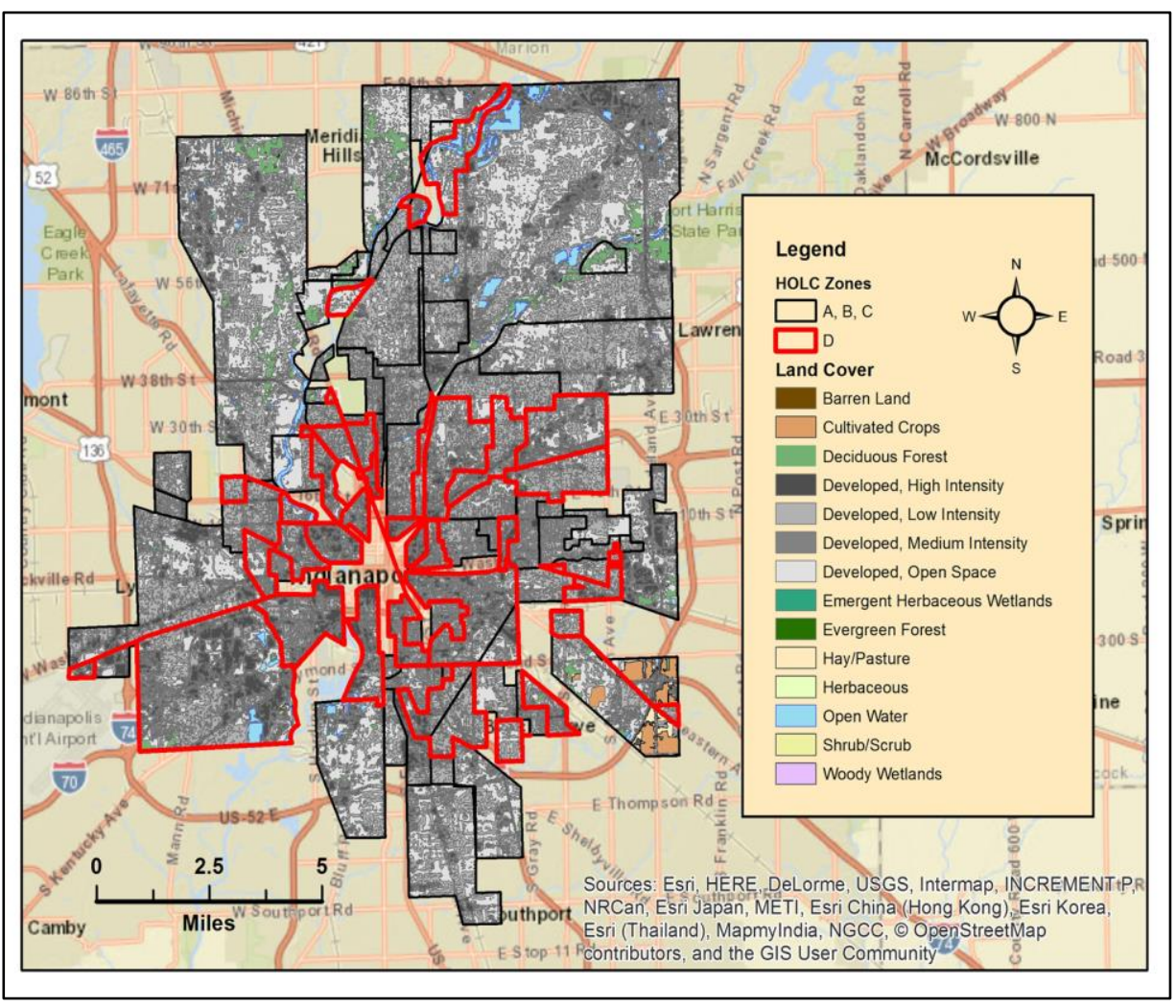

Figure 5. Land cover (2011) as it was distributed through the lending security zones of the HOLC lending security map (1937). Zones that were "redlined," where residents were least likely to be accepted for loans in the 1930s, are highlighted. 


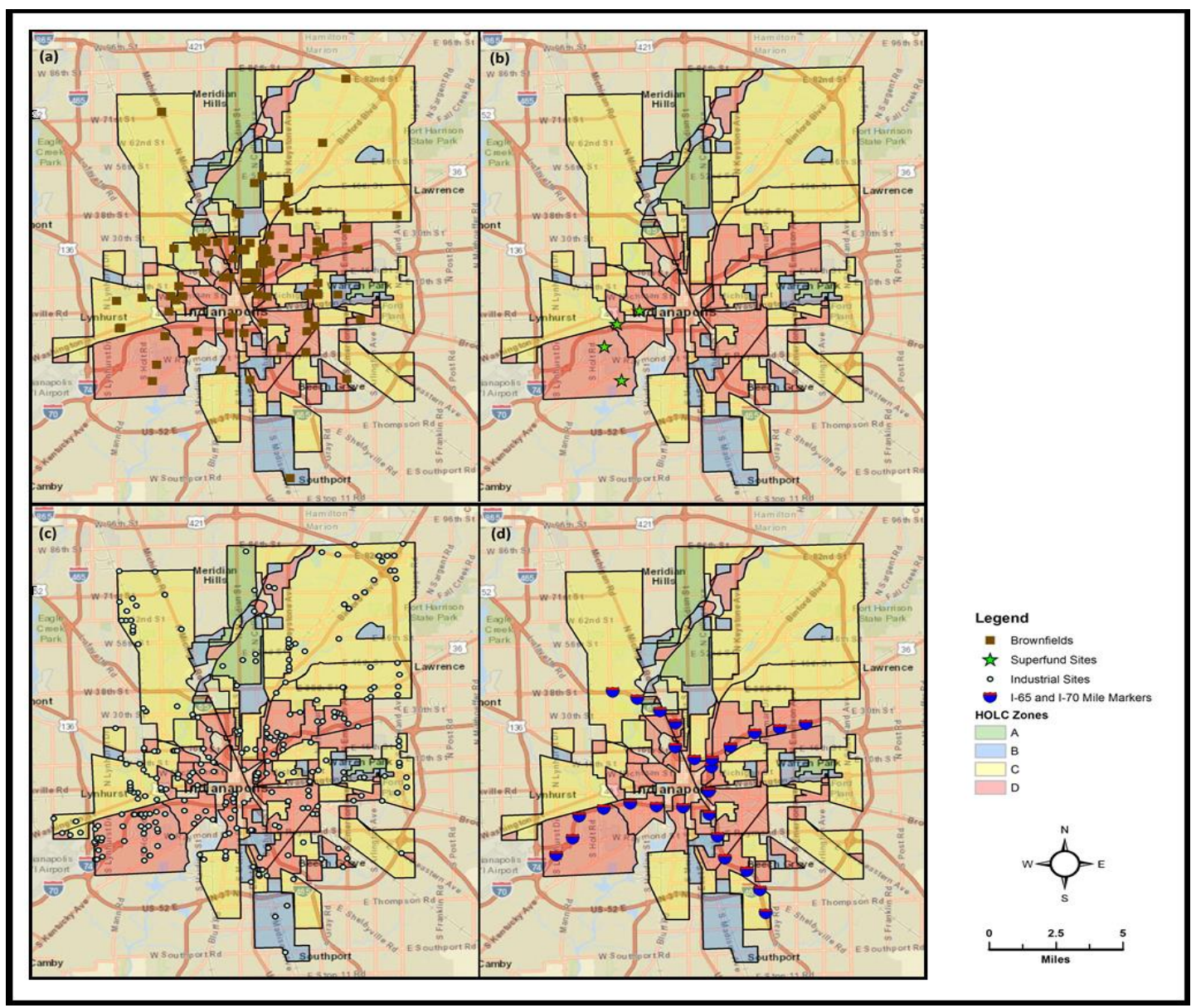

Figure 6. Distribution of modern attributes throughout the zones designated in the HOLC lending security map (1937). Mapped are (a) brownfields, (b) Superfund sites, (c) industrial sites, and (d) interstate highway (I-65 and I-70) mile markers. (Sources: IDEM 2015a, IDEM 2015b, IDEM 2015c, INDOT 2015).

cover, thus limiting the extent of direct comparison that could be made. Use of higher-resolution imagery, such as the 1-meter raster produced by the National Agriculture Imagery Program (NAIP), could make estimations and recommendations more precise. Recall from the Methods that a failed attempt was made to display vegetative land cover more precisely using a Normalized Difference Vegetation Index (NDVI) technique. This method, which involves performing a Map Algebra process on separate red and near-infrared bands of aerial imagery, should be revisited in future study by stakeholders possessing a need for such precision and the computing power to deliver it. For instance, a more thorough canopy cover analysis that detects lower-density tree plantings - such as street trees planted in single-file rows - becomes possible using a raster layer with higher resolution and could inform policy for an urban forest program operating on smaller scales. Alternately, this article demonstrates that a more practical approach might be to complete land cover analyses with a 30meter raster, as NLCD data could be more easily accessed and require less manipulation using a GIS.

Trees contribute ecosystem services at a variety of spatial scales. At the individual level, a tree provides for shade, carbon and water exchanges with the environment, and management of runoff. At the neighborhood or city levels - scales to which the 30-meter resolution applies - detectable tree cover should provide these same benefits and more. Thermal regulation, support for wildlife diversity, energy savings from shade, and reduced runoff and pollution of water are provided for when streets are lined with trees. Scaled up to the level of the whole city, greater urban forest canopy cover plays a role in reducing runoff, particulate pollution, and urban heat island effects (Livesley et al. 2016).

Preserving existing forest patches and managing land to encourage the growth of such patches should be considered a priority throughout the city, regardless of redlining history. The trends evident from this initial analysis, however, suggest that extra efforts might be dedicated to tree planting and conservation efforts in the historically redlined zones. By the measure employed in this analysis, 
equitable distribution of tree canopy benefits may not be realized unless urban development is considered a reversible process. Patches of developed land that are open spaces, making up $19 \%$ or more of the modern land cover across all historical HOLC grades (Table 2), need to be considered for their potential for conversion to forested or other vegetative land cover as an alternative to intensifying development.

Increasing forest cover may also be an appropriate goal for environmentally burdened plots of land. The point features mapped in Figure 6 bring attention to neighborhoods, historically redlined or not, which potentially stand to gain the most from tree planting and other forms of reinvestment today. Threats to human health or habitat can be inferred by these existing data, but much remains to be explored in finer detail. For instance, quantifying and mapping the areal extent of Indianapolis brownfields, estimated toxicity and history of Superfund site threats, the quantities and chemical properties of industrial waste, and traffic flow patterns of the cross-cutting interstates could add depth to the understanding of their ecological impacts on the city and its inhabitants.

Any problems revealed by this type of analysis have built in complexities, both inherent to urban social-ecological dynamics and unique to recognizing and addressing troubling correlations between variables separated by multiple generations. Accordingly, solutions must be developed through novel collaborations of governmental and nongovernmental actors. The task of identifying and illustrating these legacies can and should be accomplished by diverse researchers, and the protocol in this article was designed with this in mind. Researchers of various backgrounds and/or modest education levels should be able to see if and how their modern communities are shaped by historical phenomena like redlining.

\section{CONCLUSION}

Any urban setting will feature interactive social and ecological factors and, in this project, ecological features within social patches were explored. Modern social factors may reveal a different type of legacy, and a social scientist could bring demographic, governmental, or infrastructural data into the analysis. Layers within each of these categories can be found among the IndianaMAP resources and, consequently, would be available for comparative study of Indiana's redlined cities. Likewise, Indiana cities might be compared on the basis of any of the ecological factors included in this project. Other ecological layers may be considered, as informed by the literature and/or made available through other sources. While most of the ecological data layers available from IndianaMAP are comprised of data from recent years, the addition of any historical ecological data or observations would help advance and contextualize understanding of the ecological legacies that are already apparent.

Depending on emergent research questions, data deemed relevant might vary. For example, Reilly Tar and Chemical, one of the four point features in the Superfund dataset, could be considered for more than its name, location, and Superfund-listed status. For instance, wood treatment and coal-tar refining were performed at Reilly from the 1950s until 1972, the intense damage of their practices was largely undiscovered until the 1980s, and the resource most threatened by the site is groundwater (Fuller 2015). The challenge or cost of collecting some data may be prohibitive, but if more complex questions are posed, existing datasets might be expandable to meet updated needs.

Overall, the strengths of this method are in its relative simplicity and convenience. Few spatial analysis steps were required to take the analysis to its present state, and all data were freely available within the state of Indiana. The method will be improved as more varied and/or detailed datasets can be incorporated, both of social and ecological natures, with more time and collaboration. On the shorter term, a database for comparing all redlined cities in the state or nation could be built from analyses like this one if the technique can be optimized with respect to features of interest, standardization of methods, availability of GIS software, and accessibility of data. Then, numerous professional and student GIS analysts could divide the work involved, and this corner of the research community could move onto comparative studies more quickly 
than just one to a few researchers and could even build layers and collect data for all redlined cities. For any given researcher or team, state- or regionallevel comparisons should be realistic on the short term and larger analyses can organically build upon these if collaborative work is emphasized.

\section{ADDENDUM}

While in revision, the authors learned the University of Richmond site responsible for providing digital scans of redlining maps for U.S. cities recently updated its collection with new resources which could accelerate the type of redlining legacy analysis performed for this article. For each of the redlined metropolitan areas, including Indianapolis, the updated page provides a georectified scan and GIS-ready (Shapefile or GeoJSON) files for download (Nelson et al. 2019).

\section{LITERATURE CITED}

Cooper, G., Liberti, A., Asch, M. 2016. Replanting Durham's Urban Forest. Final Master Project, Nichols School of the Environment. Duke University.

Fuller, T. 2015. Environmental Justice and Activism in Indianapolis. Lanham, MD: Lexington Books.

Hillier, A.E. 2015. Redlining and the Homeowners' Loan Corporation. Journal of Urban History 29(4): 394-420.

Indiana Department of Environmental Management (IDEM). 2015a. BROWNFIELDS_IDEM_IN: Brownfield Locations in Indiana. Map. 1:24,000.

Indiana Department of Environmental Management (IDEM). 2015b. SUPERFUND_IDEM_IN: Superfund Program Facilities in Indiana. Map. 1:24,000.
Indiana Department of Environmental Management (IDEM). 2015c. WASTE_INDUSTRIAL_IDEM_IN: Industrial Waste Sites in Indiana. Map.

Indiana Department of Transportation (INDOT). 2015. MILE_MARKERS_SYSTEM1_INDOT_IN.SHP: MileMarker Reference Post Inventory for Roads in Indiana. Map.

Livesley, S.J., McPherson E.G., Calfapietra, C. 2016. The Urban Forest and Ecosystem Services: Impacts on Urban Water, Heat, and Pollution Cycles at the Tree, Street, and City Scale. Journal of Environmental Quality 45: 119124.

Nelson, R.K., Winling L., Marciano R., Connoly, N. Mapping Inequality. American Panorama, ed. R.K. Nelson and E.L. Ayers. https://dsl.richmond.edu/panorama/redlining/\#loc=5/45.19 8/-96.68\&text=downloads

United States Census Bureau. 2015. QuickFacts Indianapolis City (balance), Indiana. https://www.census.gov/quickfacts/table/RHI805210/1836 003

United States Environmental Protection Agency (US EPA). Superfund Site Search. https://www.epa.gov/superfund/search-superfund-siteswhere-you-live

United States Geological Survey (USGS). 2015. LAND_COVER_2011_USGS_IN: Land Cover in Indiana. Map. 1:24,000.

University of Richmond. Mapping Inequality: Redlining in New Deal America. https://dsl.richmond.edu/panorama/redlining/ 